\title{
Quantitative Evaluation of Leaf Inclination Angle Distribution on Leaf Area Index Retrieval of Coniferous Canopies
}

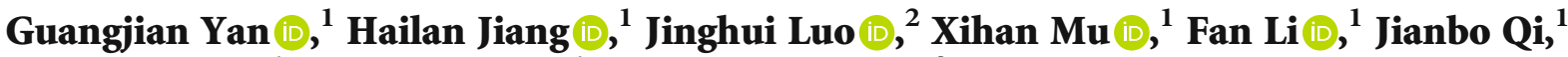 \\ Ronghai Hu $\mathbb{D}^{1},{ }^{1}$ Donghui Xie $\mathbb{D}^{D},{ }^{1}$ and Guoqing Zhou $^{3}$ \\ ${ }^{1}$ State Key Laboratory of Remote Sensing Science, Faculty of Geographical Science, Beijing Normal University, Beijing 100875, China \\ ${ }^{2}$ Southwest China Research Institute of Electronic Equipment, Chengdu 610036, China \\ ${ }^{3}$ Guilin University of Technology, Guilin 532100, China
}

Correspondence should be addressed to Jinghui Luo; wsljh555@163.com

Received 2 December 2020; Accepted 3 March 2021; Published 12 April 2021

Copyright @ 2021 Guangjian Yan et al. Exclusive Licensee Aerospace Information Research Institute, Chinese Academy of Sciences. Distributed under a Creative Commons Attribution License (CC BY 4.0).

Both leaf inclination angle distribution (LAD) and leaf area index (LAI) dominate optical remote sensing signals. The G-function, which is a function of LAD and remote sensing geometry, is often set to 0.5 in the LAI retrieval of coniferous canopies even though this assumption is only valid for spherical LAD. Large uncertainties are thus introduced. However, because numerous tiny leaves grow on conifers, it is nearly impossible to quantitatively evaluate such uncertainties in LAI retrieval. In this study, we proposed a method to characterize the possible change of G-function of coniferous canopies as well as its effect on LAI retrieval. Specifically, a Multi-Directional Imager (MDI) was developed to capture stereo images of the branches, and the needles were reconstructed. The accuracy of the inclination angles calculated from the reconstructed needles was high. Moreover, we analyzed whether a spherical distribution is a valid assumption for coniferous canopies by calculating the possible range of the G-function from the measured LADs of branches of Larch and Spruce and the true G-functions of other species from some existing inventory data and threedimensional (3D) tree models. Results show that the constant $G$ assumption introduces large errors in LAI retrieval, which could be as large as $53 \%$ in the zenithal viewing direction used by spaceborne LiDAR. As a result, accurate LAD estimation is recommended. In the absence of such data, our results show that a viewing zenith angle between 45 and 65 degrees is a good choice, at which the errors of LAI retrieval caused by the spherical assumption will be less than $10 \%$ for coniferous canopies.

\section{Introduction}

Leaf area index (LAI) is an important parameter of vegetation canopy structure and is critical in the quantitative calculation of global mass and energy exchange between the biosphere and the atmosphere $[1,2]$. Beer's law [3] is widely used to retrieve LAI from gap fraction. An important factor that highly affects the retrieval accuracy of LAI based on Beer's law is the leaf projection coefficient (G-function). The Gfunction is the effective projection proportion of leaves along the observing directions [4] and is dependent on the leaf inclination angle distribution (LAD) (assuming a uniform azimuth angle). Previous studies showed that LAD could be highly variable depending on a number of factors such as tree species, light exposure, growing season, and canopy height [5-9]. However, due to the inconvenience of in situ measurements, the spherical distribution assumption $(G \equiv 0.5)$ of
LAD is often used in LAI retrieval [10-13]. Some groundbased measurements utilize the gap fraction data close to a view zenith angle of $57.5^{\circ}$, where the values of the G-function with various LADs are close to 0.5 [14-17]. However, assuming G to be 0.5 may introduce large errors to the retrieval of LAI in other view directions.

The LAD measurement methods can be classified into two categories: direct and indirect. Direct methods measure leaf inclination angles by direct contact. A protractor with a lead hammer, an inclinometer, or a digitizer is used to measure the inclination angles of a certain number of leaves $[18,19]$. These methods are time-consuming, labor-intensive, and fraught with potential errors [20, 21]. Indirect methods can be divided into several types: (i) a digital photography measurement was proposed by Ryu et al. (2010) [22]. It uses a horizontal digital camera to view canopies and then manually measure the inclination angles of leaves 
which are approximately perpendicular to the viewing direction of the camera. This method was used in many studies and was then applied to Unmanned Aerial Vehicles (UAV) platforms [9, 23-25]. However, a large amount of humancomputer interactions are required, and it is difficult to measure the leaf inclination angles on tall forest canopies. (ii) Another method was proposed to derive leaf inclination angles based on the relationship between light transmittance and LADs by using Beer's law under the assumption of randomly distributed leaves [26, 27]. However, this method only provides the statistical average of leaf inclination angles rather than detailed LAD. In addition, the accuracy is affected by the nonrandom distribution of leaves and light conditions. (iii) With the development of Light Detection And Ranging (LiDAR) techniques, the use of Terrestrial Laser Scanning (TLS) has become more prevalent [21, 28-32]. However, it is difficult to extract individual needles from the LiDAR point clouds. As a result, current TLS-based methods mainly focus on the broad-leaved trees. (iv) Stereo imaging, which can be used to reconstruct vegetation canopies with photos taken from two or more positions based on the principle of geometric perspective, is a suitable approach to extract LAD. Wang et al. (2009) [33] reconstructed a three-dimensional (3D) corn model with photos and measured its LAD. MüllerLinow et al. (2015) [34] derived 3D surface models of plants from stereo images taken by two cameras aligned in a fixed geometry and computed geometric properties from them. Mobile phone photography is more portable and efficient compared with using the professional cameras. Qi et al. (2019) [35] proposed a new method to reconstruct 3D models of leaves using multiangle mobile photos that can be used to calculate accurate LADs.

The above methods are suitable mainly for broadleaf canopies. Convenient and efficient methods for measuring LADs for coniferous canopies have been less studied. Inspired by the method proposed by Müller-Linow et al. (2015) [34], we developed a portable and inexpensive measurement equipment called Multi-Directional Imager (MDI) that can capture stereo images of canopies, from which the leaf inclination angles can be computed. 3D line segment models were generated from stereo images taken from multiple directions, based on line segment detector and line-based 3D reconstruction method. Line segments can be used as image features to reconstruct the 3D scene [36]. Some methods have been proposed using the pure line-based Structure-from-Motion (SfM) approach, which is challenging for pose estimation [37-39]. Camera pose information was used to overcome this problem by some subsequent Multiview Stereo (MVS) approaches (e.g., SURE [40]). However, MVS methods are typically accompanied by high computational costs. In this paper, we extracted 3D models with a low computational costs algorithm called Line3D++, developed by Hofer et al. (2015) [41].

In addition to the LAD, another factor that greatly affects the Beer's law-based LAI retrieval is the clumping effect, which is caused by the nonrandomly spatial distribution of leaves in the canopy $[1,42]$. Few studies both correct the clumping effect and calculate the true G-function in LAI retrieval [43]. The clumping effect usually occurs at different spatial scales [44], including the large gaps-induced between- crown clumping, crown-shape clumping, and within-crown clumping due to the nonrandomness of foliage in the crown; these are difficult to be quantified. Thus, the clumping effect is not in the scope of this paper. Directly applying Beer's law under the assumption of a randomly spatial distribution of leaves, we can obtain the effect of LAI. Given that measuring the angles of all the needles of a tree is challenging, we explored how to obtain the possible LADs and range of Gfunction of our field-measured species. To expand the calculation of LAD and G-function on more trees with various species, we further used existing inventory data and 3D tree models. Our objective was to explore the possible effect of LAD on effective LAI retrieval for coniferous canopies, which we achieved by the following:

(1) Developing a method for retrieving LAD for coniferous branches using MDI

(2) Obtaining the possible range of the G-function for the canopies of the studied Spruce and Larch species

(3) Calculating LADs and G-functions of other species based on existing inventory data and 3D tree models

(4) Analyzing the possible theoretical impact of LAD on Beer's law-based LAI retrieval

\section{Materials}

Normally, the leaf area of a single needle is far smaller than that of a broad-leaved tree. It is difficult to distinguish needles in field measurement, even with the use of high-resolution digital cameras. Hence, we selected typical branch samples and measured the LADs of their needles in the laboratory. Numerous measurements were carried out to calculate the possible LADs and the range of G-function.

\subsection{Field Measurement Using Multi-Directional Imager}

2.1.1. Multi-Directional Imager. MDI (Figure 1) was developed based on our Multispectral Canopy Imager (MCI) [45]. The improvement included upgrading three high-resolution digital cameras and adding a synchronous exposure device, which is useful to overcome the possible movements of the samples. MDI is a low-cost and portable instrument and can be used to obtain canopy images from multiple perspectives simultaneously without the help of any direction marker.

MDI consists of the following components: (i) three digital cameras (Canon 750D) with a suite of zoom lenses (EF-S 18-135 mm f/3.5-5.6 IS STM) per set. The CMOS size of each camera is $22.3 \mathrm{~mm} \times 14.9 \mathrm{~mm}$, and the maximum resolution is $6000 \times 4000$ per camera; (ii) a platform and supporting metal arms. The platform, which can be rotated from $0^{\circ}$ to $360^{\circ}$ in the azimuth direction and $0^{\circ}$ to $90^{\circ}$ in the zenith direction, is designed for controlling the camera's orientation arbitrarily; (iii) a set of exposure controllers, which is used to control the simultaneous exposure of the three cameras; (iv) a tripod, which is used to support the platform and the cameras equipped on it.

Before the experiment, calibration is needed to obtain the interior orientation parameters of the cameras for the 


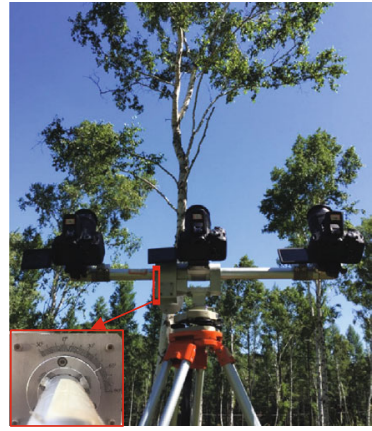

(a)

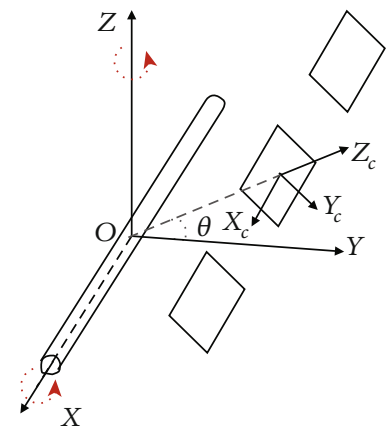

(b)

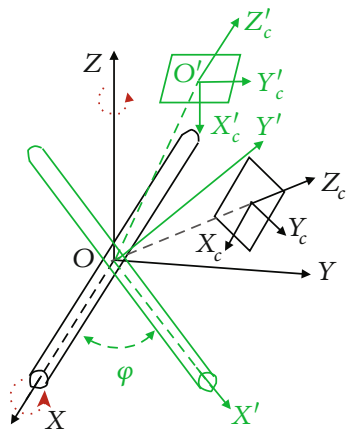

(c)

Figure 1: (a) Multi-Directional Imager (MDI). (b, c) A schematic diagram of the MDI system, where (b) and (c) represents coordinate transformation in zenith and azimuth direction, respectively.

accurate measurement of the leaf inclination angles. The calibration of the MDI measuring system mainly consists of two parts: the calibration of the platform and that of the cameras. The platform was manufactured by a professional company and had been calibrated before use. The camera calibrations were carried out in the laboratory by using a checkerboard. Table 1, which can be used as a reference for selecting the camera focal length in the experiments, summarizes the theoretical maximum pixel size in an image when the actual observation distance is $10 \mathrm{~m}$ for two different camera resolutions $(6000 \times 4000$ and $1920 \times 1280$, respectively $)$.

2.1.2. Study Sites and Samples. Field measurement campaigns of LADs were carried out from July 21 to July 26, 2016 , in Chengde Saihanba National Forest Park $\left(42.3^{\circ} \mathrm{N}\right.$, $117.2^{\circ} \mathrm{E}$ ), including the local sampling of several conifer branches (Table 2): four Larch samples and three Spruce samples (see Section 4.2 for more details). There are some differences in measurement between Larch and Spruce samples: (i) for Larch sample I and II, the samples were fixed but the direction of the MDI observation changed; (ii) for Larch sample III and IV, the branches were rotated in different orientations but the MDI was fixed; (iii) for Spruce samples, both the samples and the direction of the MDI observation were fixed. This was done to test whether the direction of the MDI observation and the rotation of the branches have some influence on LAD and G-function (Details in section 4.3). In Table 2, the underlined sample ID denotes different observing directions of MDI around a specific sample; the corresponding number of photos taken by MDI are listed. In the experiment, the branch samples were scissored at different crown heights from a few representative trees. The branch samples were measured in the laboratory in one day after cutting to prevent the atrophy of the needles. The lengths of branches were measured, and the number of needle clusters and the number of needles in each cluster were counted. A white cloth was used as the background when taking photos in the laboratory to reduce the impact of background on object detection in photography.

2.2. 3D Tree Models and Inventory Data. What we did in section 2.1 is to obtain the possible LADs and range of G- function of Larch and Spruce through rotation of the branches. To investigate more species, we further used other kinds of data: (i) 3D tree models: 71 coniferous tree models of 13 species from OnyxTREE CONIFER library (http://www .onyxtree.com/conifer.html) with known true LADs (see Table 3); (ii) inventory data: 23 trees of Järvselja Scots Pine (https://rami-benchmark.jrc.ec.europa.eu/HTML/RAMI-IV/ EXPERIMENTS4/ACTUAL_CANOPIES/JARVSELJA_SUMMER_ PINESTAND/JARVSELJA_SUMMER_PINESTAND.php) and Ofenpass Mountain Pine (https://rami-benchmark.jrc.ec .europa.eu/HTML/RAMI-IV/EXPERIMENTS4/ACTUAL CANOPIES/OFENPASS_WINTER_PINESTAND/OFENPASS WINTER_PINESTAND.php) from RAMI IV. The measured LADs of these trees, one species measured in summer and the other in winter, are provided. More details about the inventory data can be found on the RAMI IV website. The number of trees, the average, and the range of tree heights for each species are summarized in Table 3. Front views of some of the studied 3D tree models from OnyxTREE are shown in Figure 2 as examples.

\section{Method}

\subsection{Estimation of Leaf Inclination Angle Distribution}

3.1.1. Detection and Reconstruction of Needles. The exact leaf inclination angles can be calculated from 3D leaf models. LSD [46] and Line3D++ [41] were used to generate 3D line segment models from stereo images of coniferous branches. To be specific, LSD was used to extract 2D lines from each image, and Line3D++ was used to develop 3D line models.

LSD is an efficient line segment detector that can be used to acquire accurate subpixel results [47]. It follows the method proposed by Burns et al. (1986) [48] and uses a contrarian validation approach proposed by Desolneux et al. (2000) [49] to control false detections. LSD algorithms can be roughly divided into three steps. First, the image is segmented into a series of small continuous regions with the same gradient angle. Then, the most approximate line segment is determined in each successive small region. Finally, a validation criterion is used to control the number of false detections. 
Table 1: Pixel sizes of the camera under different focal lengths.

\begin{tabular}{|c|c|c|c|c|c|c|c|c|c|c|}
\hline Camera resolution & & & $0 \times 4$ & & & & & $20 \times 1$ & & \\
\hline Focal length (mm) & 18 & 35 & 50 & 85 & 135 & 18 & 35 & 50 & 85 & 135 \\
\hline The maximum physical size represented by a pixel (mm) & 2.1 & 1.1 & 0.7 & 0.4 & 0.3 & 6.5 & 3.3 & 2.3 & 1.4 & 0.9 \\
\hline
\end{tabular}

TABLE 2: Measurements of inclination angles of needles on Larch and Spruce branches.

\begin{tabular}{lcccccccccccccccccc}
\hline $\begin{array}{l}\text { Species } \\
\text { Samples }\end{array}$ & I-a11 & I-a12 & I-a13 & I-a14 & II-a21 & II-a22 & $\underline{\text { II-a23 }}$ & II-a24 & III-a31 & III-a32 & IV-a41 & IV-a42 & IV-a43 & a1 & a2 & a3 \\
\hline No. of photos & 32 & 18 & 10 & 30 & 16 & 8 & 18 & 54 & 18 & 14 & 16 & 23 & 23 & 15 & 23 & 15 \\
\hline
\end{tabular}

TABLE 3: Three-dimensional (3D) coniferous tree models and trees from RAMI IV.

\begin{tabular}{|c|c|c|c|c|c|}
\hline Species & Number of trees & Average tree height $(\mathrm{m})$ & Minimum tree height $(\mathrm{m})$ & Maximum tree height $(\mathrm{m})$ & Source \\
\hline Abies & 6 & 2.34 & 1.53 & 2.83 & \multirow{13}{*}{$3 \mathrm{D}$ tree model } \\
\hline Cedrus & 5 & 6.08 & 3.71 & 8.68 & \\
\hline Cephalotaxus & 1 & 1.81 & 1.81 & 1.81 & \\
\hline Cryptomeria & 2 & 1.74 & 1.74 & 1.74 & \\
\hline Cypress & 3 & 2.49 & 2.47 & 2.52 & \\
\hline Jeffrey's pine & 2 & 5.54 & 5.50 & 5.58 & \\
\hline Mountain hemlock & 1 & 13.00 & 13.00 & 13.00 & \\
\hline Picea & 6 & 2.46 & 2.04 & 2.83 & \\
\hline Pinus & 33 & 4.05 & 1.60 & 10.82 & \\
\hline Podocarpus & 4 & 6.03 & 5.60 & 6.62 & \\
\hline Pseudotsuga & 2 & 2.08 & 2.07 & 2.08 & \\
\hline Taxus & 4 & 0.89 & 0.85 & 0.94 & \\
\hline Yew shrub & 2 & 1.31 & 1.28 & 1.34 & \\
\hline Järvselja Scots pine & 9 & 15.72 & 11.78 & 18.56 & \multirow{2}{*}{$\begin{array}{l}\text { Inventory } \\
\text { data }\end{array}$} \\
\hline Ofenpass Mountain pine & 14 & 9.63 & 1.09 & 15.12 & \\
\hline
\end{tabular}

Line3D++ is a novel algorithm using line segments as fundamental features to abstract 3D scenes, which consists of several steps. First, a large number of potential line correspondences are established using epipolar geometry constraints. The epipolar geometry can be computed through known camera poses, which can be obtained by running any Structure-from-Motion (SfM) pipelines. Then, the most suitable correspondence is selected for each line segment. Hofer et al. (2017) [50] used a scoring formulation composed of angular similarity and positional similarity to distinguish between correct and incorrect matches. The final 3D line model is obtained by fusing 2D line correspondences through an arbitrary graph-clustering algorithm.

Figure 3 shows an example of line segment detection and reconstruction using LSD and Line3D++ algorithms. Apart from some highly occluded regions, most of the needles are detected from the original images. The result (Figure 3(c)) shows that the majority of needles are reconstructed effectively.

3.1.2. Calculation of Leaf Inclination Angle Distribution. The data processing flow chart of the LAD retrieval is shown in Figure 3. First, MDI was used to obtain the multiangle stereo images of the branch samples (Figure 3(a)). Then, dense matching and linear feature extraction techniques were used to get the orientation of each needle automatically from the coaxial stereo image pairs obtained by MDI. Finally, the view zenith angle of MDI was used to calculate the final LAD (Figure 3(d)) based on the extracted inclination angles of the needles.

Through the above steps, we can obtain the distribution of the needles in the camera coordinate system. It has to be converted to the world coordinate system to obtain the real inclination angles of the needles. From the schematic diagram of the MDI coordinate system shown in Figure 1, $X Y Z$ represents a real-world local coordinate system. $X$ is the direction of the metal arms, and $Z$ is the zenith direction. $X_{c} Y_{c} Z_{c}$ represents the camera space coordinate system of the intermediate camera. The angles of the platform in the vertical direction ( $\theta$ in Figure 1(b)) can be read directly from the graduated collar on the rotatable platform.

$\mathbf{v}$ represents the real vertical direction, which is transformed from the world space to the camera space. The inclination of the needle in world space $\theta_{l}$ is defined as

$$
\theta_{l}=\left\{\begin{array}{ll}
\arccos \frac{\mathbf{n} \cdot \mathbf{v}}{\|\mathbf{n}\|\|\mathbf{v}\|} & \mathbf{n} \cdot \mathbf{v}>0 \\
180^{\circ}-\arccos \frac{\mathbf{n} \cdot \mathbf{v}}{\|\mathbf{n}\|\|\mathbf{v}\|} & \mathbf{n} \cdot \mathbf{v} \leq 0
\end{array},\right.
$$



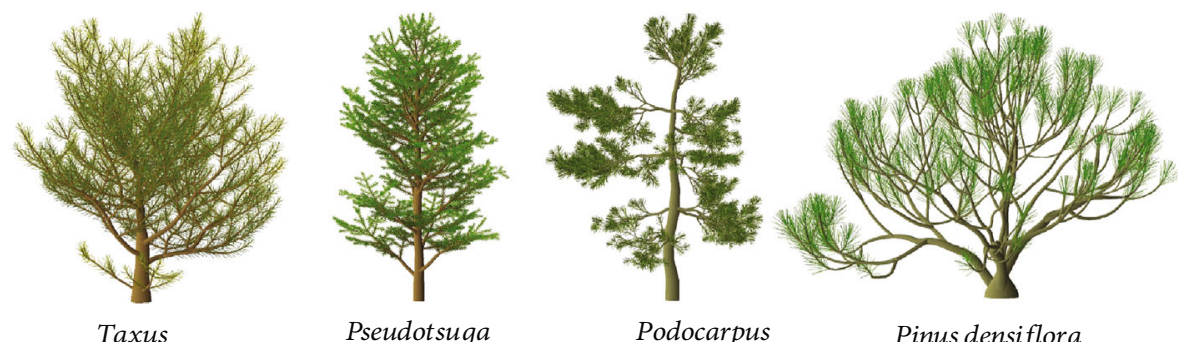

Pseudotsuga

Podocarpus
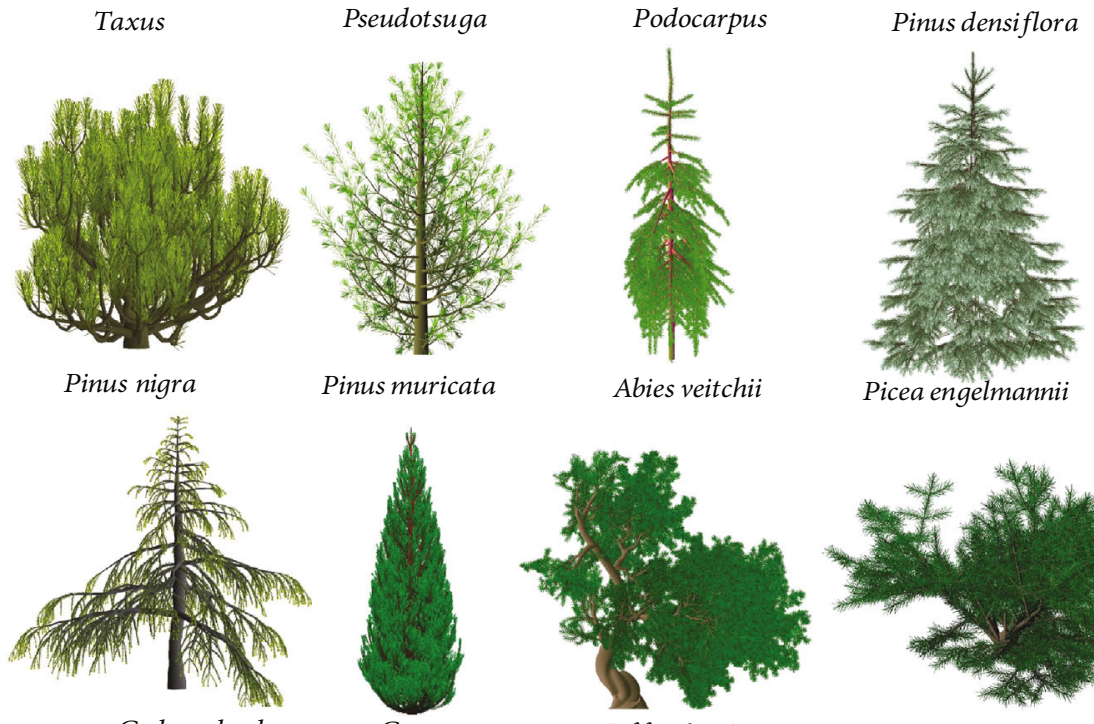

Pinus muricata

Abies veitchii
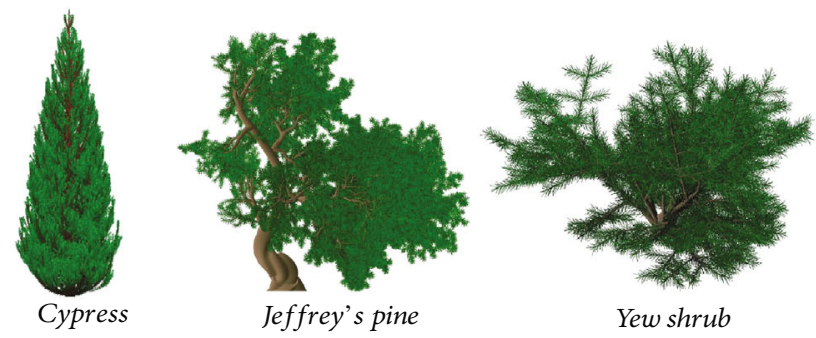

FIgURE 2: Examples of some three-dimensional (3D) coniferous tree models from the OnyxTREE CONIFER database.

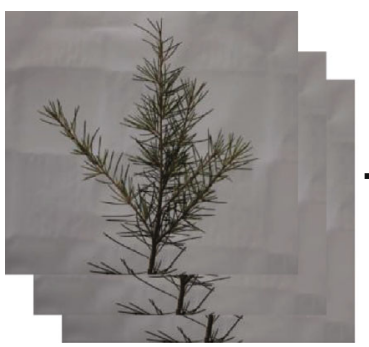

(a)

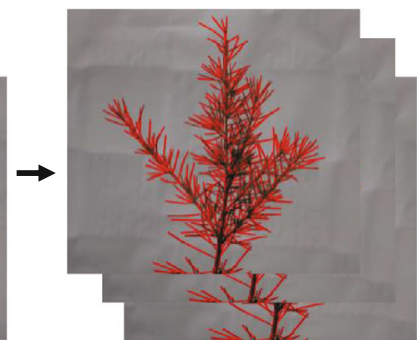

(b)

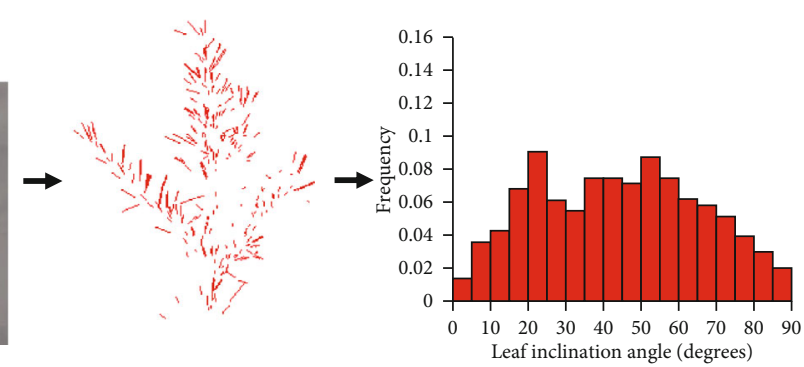

(c)

(d)

FIgure 3: Data processing flow chart for calculating the leaf inclination angle distribution (LAD). (a) Example stereo images of a needle branch sample. (b) Line segments detection using LSD algorithm. (c) Three-dimensional (3D) lines reconstruction using Line3D++ algorithm. (d) The calculation of LAD.

where $n$ indicates the normal vector of a needle in camera space, $v=(0,-\cos \theta, \sin \theta)$.

The final LAD is obtained by weighting the relative areas with different leaf inclination angles.

\subsubsection{Validation of the Retrieved Leaf Inclination Angles. To} validate the accuracy of our method, the inclination angles of needles on a coniferous tree branch were measured manually with a protractor and were assumed to be ground-truth. It is unrealistic to measure the inclination angle of each needle directly because the needles on a branch are dense and are sheltered from each other; hence, most of the needles were cut off from the branch, and only 16 remaining needles were measured manually (Figure 4(a)). Meanwhile, 3D needle models were reconstructed from the stereo images obtained from MDI, and the inclination angles were calculated automatically. Then, the coefficient of determination $\left(R^{2}\right)$ and the root mean square error (RMSE) were calculated. The results are shown in Section 4.1.

3.2. G-Function Calculation. The definition of the G-function proposed by Stenberg (2006) [51] was used in this study:

$$
G\left(\theta, \theta_{l}\right)=\frac{2}{\pi^{2}} \int_{0}^{\pi}\left[1-\left(\cos \theta \sin \theta_{l}+\sin \theta \cos \theta_{l} \cos A\right)^{2}\right]^{1 / 2} d A,
$$




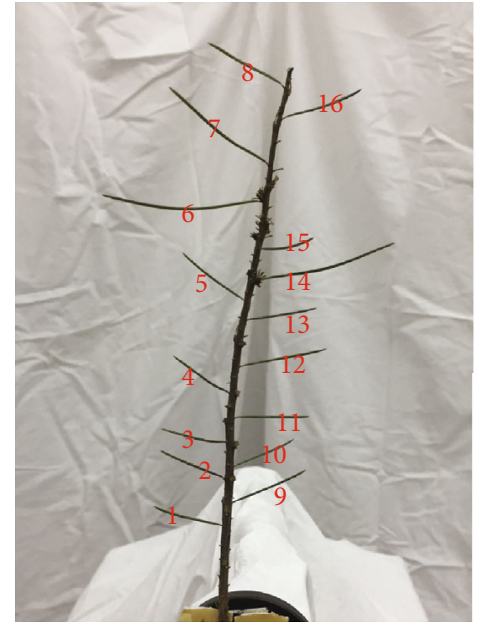

(a)

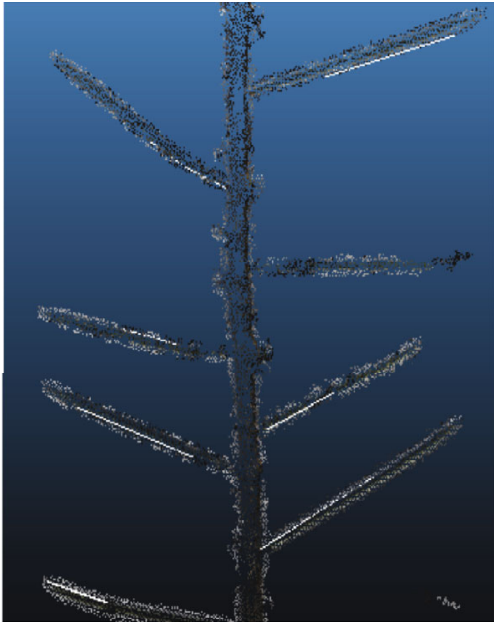

(b)

Figure 4: (a) Needle samples and (b) the partially enlarged details of automatically extracted needles (white lines) and the reconstructed point clouds.

TABLE 4: Comparison of the leaf inclination angles from manual measurements and the automatic algorithm.

\begin{tabular}{lccccc}
\hline Needle ID & Manual measurement & Algorithm extraction & Needle ID & Manual measurement & Algorithm extraction \\
\hline 1 & $18^{\circ}$ & $19.02^{\circ}$ & 9 & $26^{\circ}$ & $24.70^{\circ}$ \\
2 & $26^{\circ}$ & $26.01^{\circ}$ & 10 & $12^{\circ}$ & $-71^{\circ}$ \\
3 & $19^{\circ}$ & $17.66^{\circ}$ & 11 & $3^{\circ}$ & $12.79^{\circ}$ \\
4 & $35^{\circ}$ & $36.88^{\circ}$ & 12 & $12^{\circ}$ & $12.60^{\circ}$ \\
5 & $34^{\circ}$ & $32.09^{\circ}$ & 13 & $13^{\circ}$ & $15^{\circ}$ \\
6 & $10^{\circ}$ & $10.12^{\circ}$ & 14 & $12^{\circ}$ & - \\
7 & $17^{\circ}$ & $15.27^{\circ}$ & 15 & $17^{\circ}$ & $14.75^{\circ}$ \\
\hline
\end{tabular}

where $\theta$ is the view zenith angle, $\theta_{l}$ is the needle inclination angle, and $A$ is the azimuth divergence angle between the needle and the observation directions.

The measured leaf inclination angles are binned to the leaf inclination angle intervals ranging from 0 to $\pi / 2$ with a step of $5^{\circ}$. Thus, the relationship between the discrete leaf inclination angle and the G-function is

$$
G(\theta)=\sum_{i=1}^{N} f_{i} \bullet G_{i}\left(\theta, \theta_{l i}\right)
$$

where $N$ is the total number of leaf inclination angle intervals and $N=18, \theta_{l i}$ is the center needle inclination angle of each interval, $f_{i}$ is the leaf area fraction of the needle inclination angle interval centered at $\theta_{l i}$, and $G_{i}\left(\theta, \theta_{l i}\right)$ is the value of the $\mathrm{G}$-function when the needle inclination angle equals to $\theta_{l i}$.

3.3. Calculating the Possible Range of G-Function of the Canopy. For the field measured species including Larch and Spruce, it is impossible to measure every needle on a tree due to the huge number of needles and highly complex canopy background. Thus, what we can obtain is just the LAD of a certain branch, not that of a tree. However, we believe that the possible range of LAD and G-function of a tree can be obtained by rotating the branches to different orientations, assuming that a tree is made up of branches in different directions. Thus, the reconstructed $3 \mathrm{D}$ needle leaf models were rotated in many different orientations in the $3 \mathrm{D}$ space to represent possible orientations of needles and branches in the real canopy, and then, the corresponding changes in LAD and G-function were studied. To obtain the 3D needle leaf models with different orientations, we used CloudCompare (http://cloudcompare.org) to rotate each reconstructed 3D needle leaf models in fifteen different orientations. The LAD and G-function were subsequently computed using the rotated 3D needle leaf models. At last, all of the possible G-functions of the Larch and Spruce samples were calculated, which can be treated as possible situations in real canopies of these two species. The real G-functions of the canopies should be in the range of these simulated G-functions due to the fact that more branches in different orientations in reality tend to smooth the change of the G-function. 


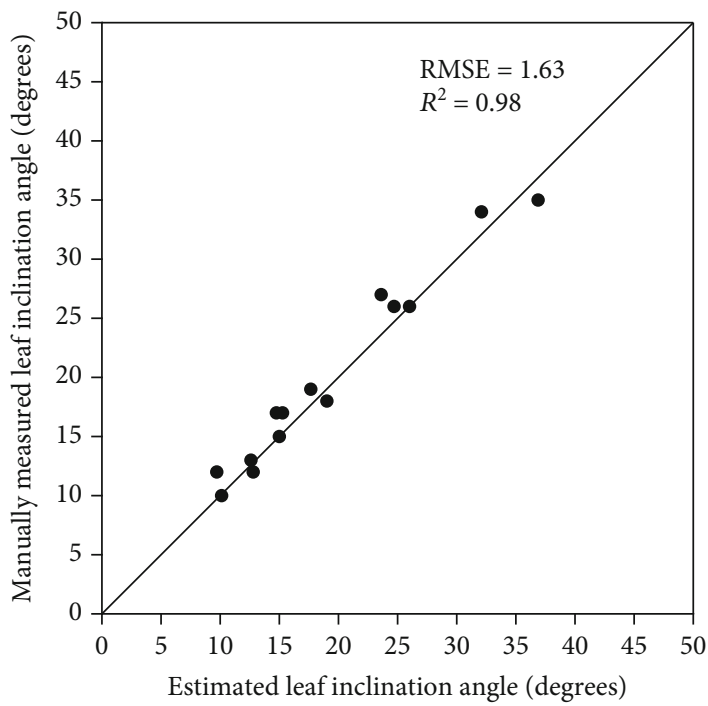

FIGURE 5: Comparison of needle inclination angles from algorithm extraction and manual measurement.

For other species from inventory data and 3D tree models, the range of G-function of the canopy is calculated directly from all the trees we collected. This is due to the fact that the $\mathrm{LAD}$ is a statistic of leaf inclination angles and is not related to spatial distribution of trees in the canopy.

3.4. Calculation of the Impact of Leaf Inclination Angle Distribution on LAI Retrieval. The traditional Beer's law of light transmission through a turbid medium is widely used in indirect LAI measurements [3,52], assuming that the medium of the vegetation is homogeneous:

$$
\begin{aligned}
P(\theta) & =\mathrm{e}^{-G(\theta) \cdot \mathrm{LAI} / \cos \theta}, \\
\mathrm{LAI} & =-\frac{1}{G(\theta)} \cdot \ln P(\theta),
\end{aligned}
$$

where $P(\theta)$ denotes the gap fraction in the viewing zenith angle $\theta ; G(\theta)$ is the G-function, assuming of spherical distribution, $G(\theta) \equiv 0.5$. LAI is the effective LAI assuming a random distribution of leaves without considering the clumping effect.

We assumed the true G-function value in view zenith angle $\theta$ as $G_{\text {true }}(\theta)$, and the relative error of LAI caused by the assumption of spherical distribution is

$$
\text { Relative error }(\theta)=\left(\frac{G_{\text {true }}(\theta)}{0.5}-1\right) \times 100 \% \text {. }
$$

From this equation, we infer that if we use the spherical distribution assumption, a $10 \%$ error in the G-function results in a $10 \%$ error in Beer's law-based LAI retrieval.

\section{Results and Discussion}

4.1. The Accuracy of Leaf Inclination Angles Retrieved from Stereo Photos. The leaf inclination angles of needles were measured manually as the ground-truth to validate the accu- racy of our proposed method. Needles on a branch (Figure 4(a)) were manually measured, and the inclination angles were calculated automatically after they were detected. The enlarged details of the automatically extracted needles and the reconstructed point clouds are shown in Figure 4(b). As what is shown in Table 4, 14 of a total of 16 needles were extracted (except needles 11 and 15).

The comparison of manual measurements and algorithm extraction is shown in Figure 5. The algorithm performs well, with an $R^{2}$ of 0.98 and a RMSE of $1.63^{\circ}$. It shows that the proposed method can be used for accurate and rapid measurement of needle leaf inclination angle.

4.2. Leaf Inclination Angle Distribution and G-Function. Both the LADs and G-functions were estimated from different conifer samples: 4 Larch samples and 3 Spruce samples. Specifically, Larch sample I and II and the Spruce samples were fixed on the cantilever brackets at the time of data acquisition, and the position of MDI was moved. Sample III and IV on the cantilever brackets were manually rotated in different orientations. Photos around the samples were taken in different directions using MDI (Figure 6(a)), and at least 8 photos (details are listed in Table 2) with good quality were selected for line segment detection and 3D needle line segment reconstruction. The final LADs were calculated from the reconstructed needles (Figure 6(b)). Different trends were found in the results. For sample I and II, there were small differences in both the LADs and the mean leaf inclination angles (MLAs) measured in different directions, indicating that the observing direction of MDI has little impact on LAD. For sample III, the difference of MLAs between a31 and a32 was small, which was probably due to the relatively close orientations of them. However, there were relatively large differences in both the LADs and the MLAs before and after rotation for sample IV, and the maximum difference of MLA was about $10^{\circ}$.

Spruce samples were fixed on the cantilever brackets when data were acquired. Photos for different samples and 

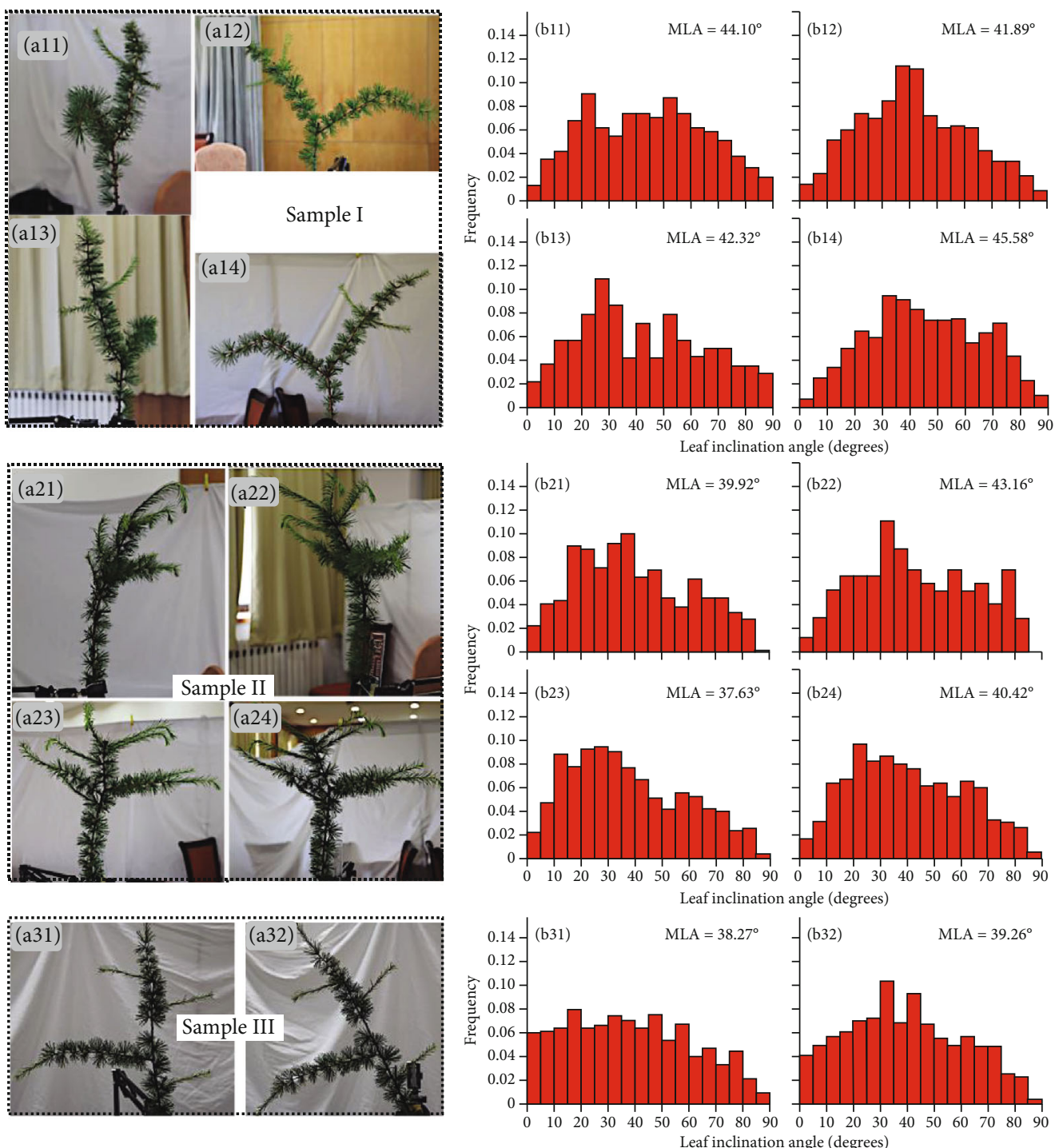

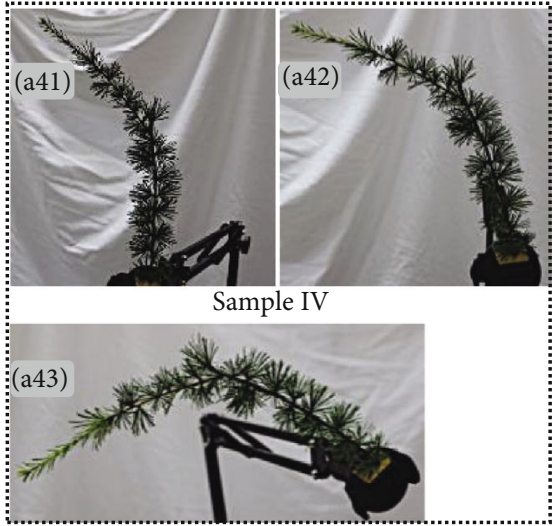

(a)

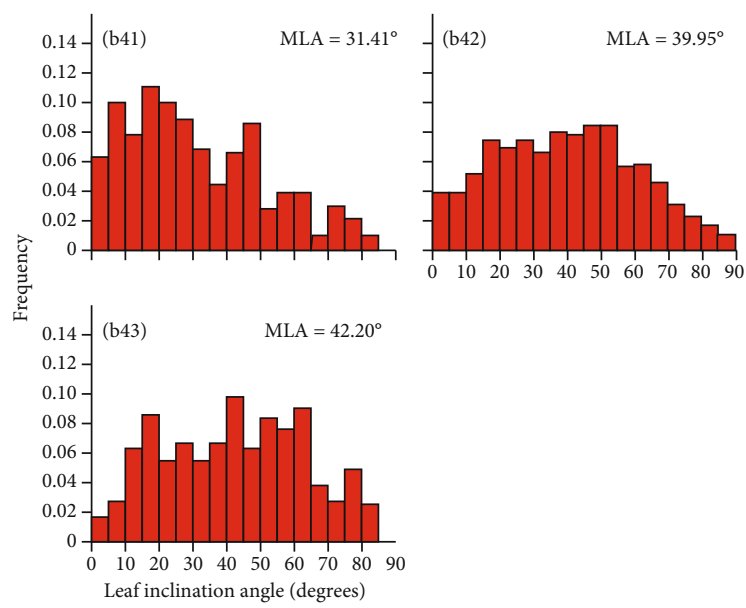

(b)

Figure 6: (a) Photos of different shoot directions of the Larch samples (see detailed information in Table 2) and (b) the measured leaf inclination angle distributions (LADs). 
the extracted LADs were shown in Figures $7(\mathrm{a} 1-\mathrm{a} 3)$ and 7(b1-b3), respectively. Results showed that the LADs might be different even with different samples of the same tree species, and the corresponding MLAs might have a large difference.

Figure 8 illustrates the G-function computed from abovementioned measurement data in this section. The G-function values calculated using the data obtained in different directions of the same conifer sample were close to each other, and the overall trend was consistent except for sample IV (black lines in Figure 8(a)). However, the G-function values of the three Spruce samples (Figure 8(b)) differ greatly.

4.3. Possible Range of the G-Function by Rotating the Reconstructed Needles. The main purpose of this section is to study the changes in LADs and G-functions with different needle orientations in the $3 \mathrm{D}$ space. We assume that the possible range of the G-function of the trees can be obtained by arbitrary rotations of the branches.

Larch sample IV-a43 was used as an example to illustrate the experimental processes and results. First, each reconstructed 3D needle leaf model was rotated in 15 different orientations in the 3D space using CloudCompare (Figure 9). We saved the rotated 3D needle leaf models and computed LADs (Figure 10) and G-functions (Figure 11).

The other remaining samples were also processed according to the above steps. Several G-functions to represent the Larch and Spruce samples in the 3D space were acquired (Figure 12). Figure 12 contains the G-function calculated from both the original reconstructed 3D needle leaf models and those after rotation.

Figures 10-12 show that large differences exist in the corresponding LADs and G-functions when the 3D needle leaf models are rotated in different orientations in the $3 \mathrm{D}$ space, which also exists in MLA. The range of Gfunctions is largest when the view zenith angle is $0^{\circ}$, which is 0.13 for Larch samples and is up to 0.21 for Spruce samples. However, the maximum range of G-function is only 0.05 and 0.09 for Larch and Spruce samples, respectively, when the view zenith angles are between $40^{\circ}$ and $70^{\circ}$, which is commonly used in field measurement campaigns. It can be inferred that changes in the values of the Gfunction are not significant in this range across the whole forest, because a real canopy has a greater number of randomly orientated branches and needles.

In addition, the overall variation range of the $\mathrm{G}$-functions of Larch samples is smaller than that of Spruce samples. The reason may lie in the different growth structure of the two different conifer species.

4.4. G-Functions of Inventory Data and 3D Tree Models. The G-functions of trees from RAMI IV inventory data and 3D tree models with known LADs were also calculated. The range of G-function is the largest when the view zenith angle is $0^{\circ}$, which is 0.09 for the inventory data (Figure 13(a)) and is up to 0.31 for the studied $3 \mathrm{D}$ tree models (Figure 13(b)). However, the maximum range of the G-function is only 0.02 and 0.09 when the view zenith angles are in $40^{\circ} \sim 70^{\circ}$ and is much smaller when the view zenith angles are in $45^{\circ} \sim 65^{\circ}$. In addition, the overall variation range of the $\mathrm{G}$-function of the inventory data is smaller than that of the $3 \mathrm{D}$ tree models, which is because both the number of trees and tree species of the 3D tree models are significantly larger than those of the inventory data, i.e., the $3 \mathrm{D}$ tree models reflect more conditions of realistic coniferous trees.

4.5. The Effects of Leaf Inclination Angle Distribution on LAI Retrieval. Due to the inconvenience of in situ measurements of LAD of coniferous trees, the spherical distribution assumption is usually used in LAI retrieval. Previous studies showed that a spherical distribution is not a valid assumption for most broadleaf tree species [53]. However, relevant research in coniferous species is still lacking. In this section, whether the spherical distribution $(G \equiv 0.5)$ is a valid assumption for coniferous canopies and the possible impact on LAI retrieval will be discussed. Specifically, we are interested in three kinds of view zenith angles: (i) $40^{\circ} \sim 70^{\circ}$, which is commonly used in field measurement campaigns; (ii) $0^{\circ}$, considering that the view zenith angle for spaceborne LiDAR instruments including Geoscience Laser Altimeter System (GLAS) and the Global Ecosystem Dynamics Investigation (GEDI) are all close to $0^{\circ}$ (up to $1^{\circ}$ for Geoscience Laser Altimeter System (GLAS) [54], and up to $6^{\circ}$ for Global Ecosystem Dynamics Investigation (GEDI) [55]); (iii) $0^{\circ} \sim 15^{\circ}$, considering that the view zenith angle for airborne LiDAR is usually less than $15^{\circ}$ and is widely used in LAI retrieval [56].

The relative errors of LAI retrieval under a constant Gfunction assumption of 0.5 were compared with those retrieved using the true G-function value for coniferous canopies (Figure 14). Results show that the relative errors of retrieved LAI depend on the view zenith angles and increase gradually with the deviation from $57.5^{\circ}$. When the view zenith angles are in $40^{\circ} \sim 70^{\circ}$, the relative errors of LAI are less than $15 \%$. When the view zenith angle is at $0^{\circ}$, LAI could be underestimated by as much as $53 \%$; when the view zenith angles range of $0^{\circ} \sim 15^{\circ}$, the relative errors of LAI are in the range of $-53 \% \sim 9 \%$. However, the relative errors are less than $10 \%$ when the view zenith angles are in the range of $45^{\circ} \sim 65^{\circ}$.

Based on the above analysis, we recommend calculating LAD accurately in LAI retrieval, especially at nadir viewing. If the exact $\mathrm{LAD}$ measurement data is absent, we recommend using the remotely sensed data captured in the zenithal angle between $45^{\circ}$ and $65^{\circ}$. In such cases, the possible LAI retrieval errors caused by fixing the Gfunction value as 0.5 is less than $10 \%$.

4.6. Limitations and Suggestions for Future Research. It is difficult to obtain a general knowledge of the effect of the LAD on LAI retrieval for coniferous canopies just based on the field measurement. This is due to the fact that the field measurement is limited to a few branches of two species, since it is challenging to measure all the branches of a tree. Thus, the LAD of a tree is hard to be obtained. Two strategies were used in this study to overcome these problems: (i) rotating branches to obtain the possible range of the G-function 

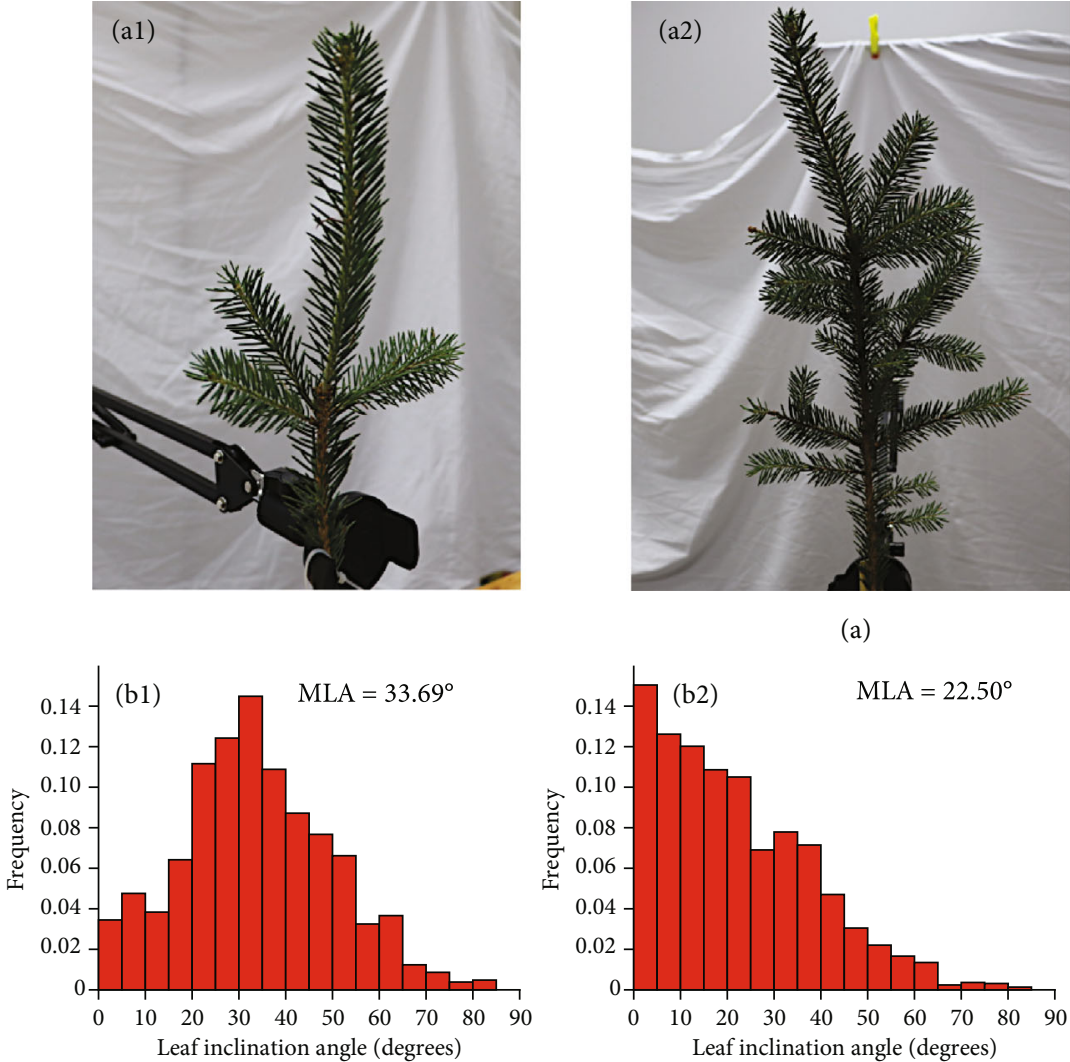

(b)
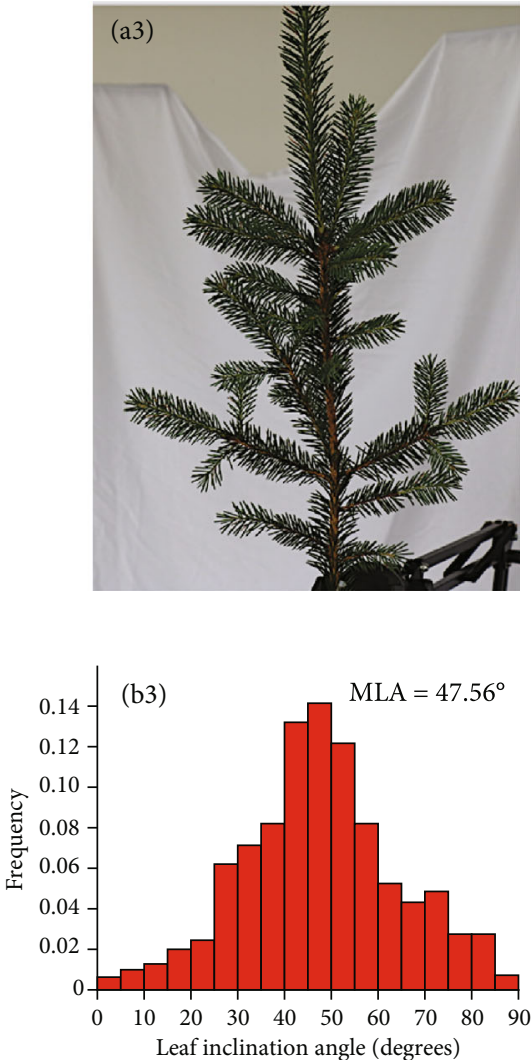

Figure 7: Photos (a1-a3) and the leaf inclination angle distributions (LADs) (b1-b3) of different Spruce samples.

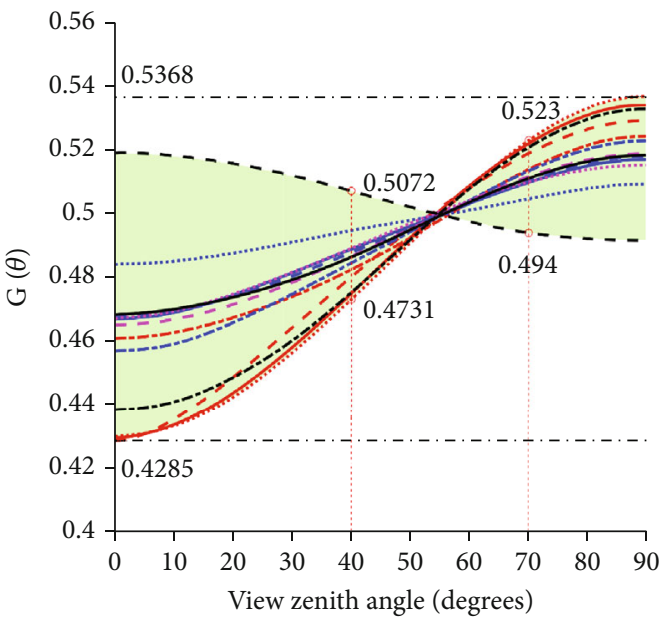

\begin{tabular}{|c|c|}
\hline I-a11 & -- II-a24 \\
\hline - - I-a12 & ...... III-a31 \\
\hline$--\mathrm{I}-\mathrm{a} 13$ & - - III-a32 \\
\hline I-a14 & -- IV-a 41 \\
\hline II-a21 & — IV-a 42 \\
\hline $\begin{array}{l}\text { II-a22 } \\
\text { II-a23 }\end{array}$ & $\cdots$ IV-a43 \\
\hline
\end{tabular}

(a)

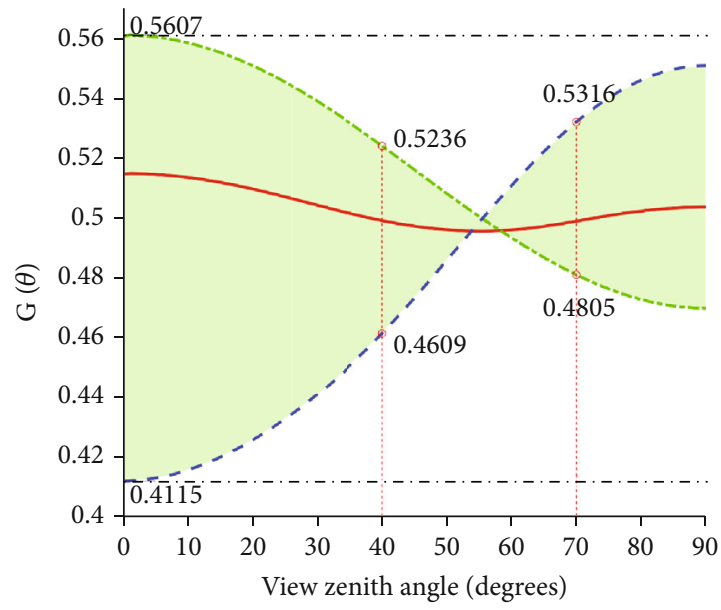

- al

$-\cdot-\mathrm{a} 2$

$---\mathrm{a} 3$

Figure 8: G-functions for (a) Larch samples and (b) Spruce samples. The light-green region represents the range of G-function contained by two horizontal dashed lines; the red dashed lines show the range of view zenith angles from 40 to 70 degrees. 

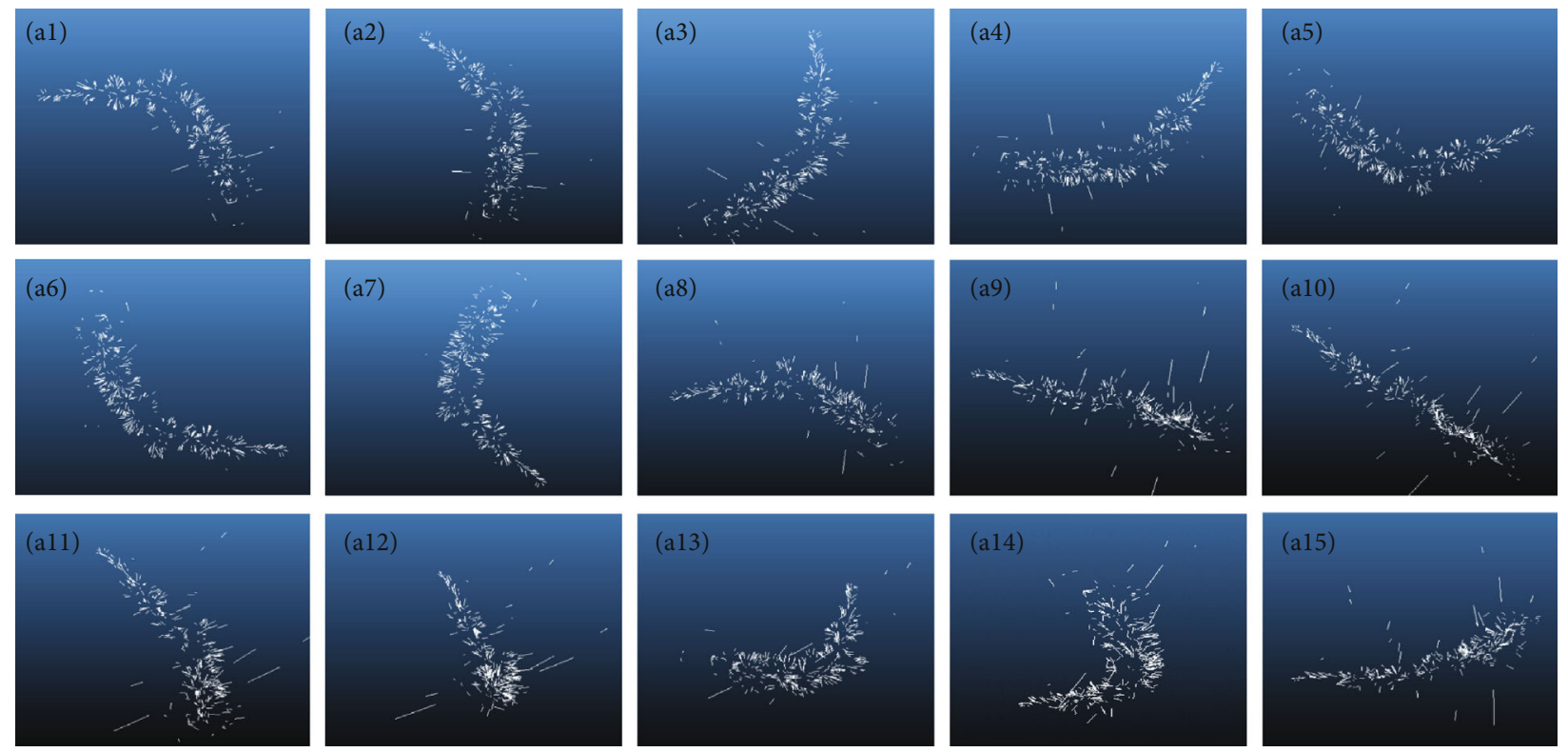

FIGURE 9: The different rotation diagrams of the reconstructed three-dimensional (3D) needle leaf models of sample IV-a43.
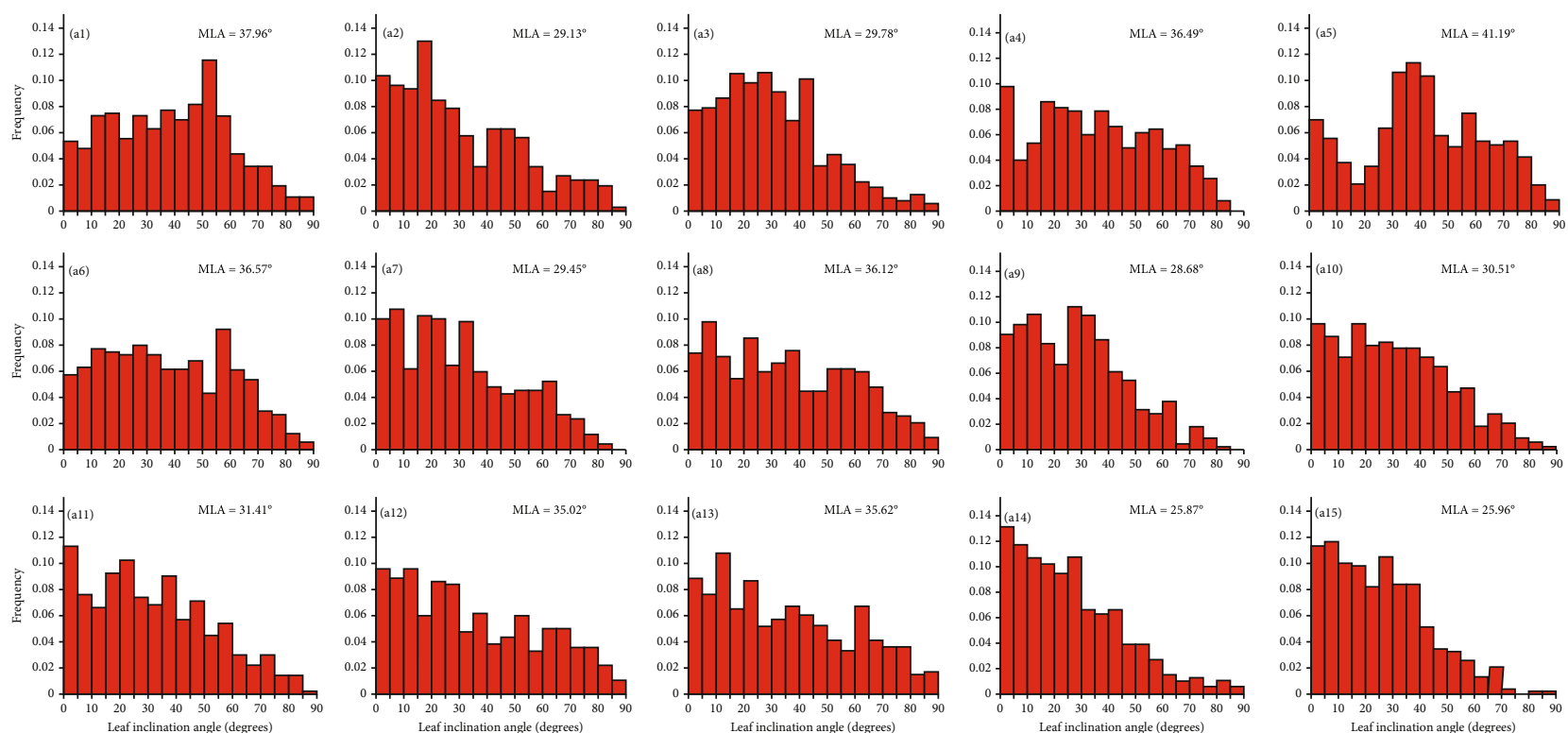

FIGURE 10: The leaf inclination angle distributions (LADs) computed from the rotated three-dimensional (3D) needle leaf models in Figure 9.

and (ii) collecting as much data as possible to find a general impact of leaf inclination angles on LAI retrieval. Inevitably, there are some limitations of this work which should be further studied:

(1) More branches can be measured in the future, considering that the selected branch samples may have some influence on the $\mathrm{LAD}$ and G-function estimation

(2) More tree models can be collected to make the finding more general
(3) More data of the same species can be collected in different seasons since the angles of leaves also change with the season. Even though both LAD data measured in summer and winter are included in the inventory data of RAMI IV, and the 71 tree models from a conifer database already contain diverse branches and leaves, which we believe can indirectly take the impact of season into consideration, a careful study on this issue is still an interesting topic

(4) Both the LAD and clumping effect can be analyzed on the influence of true LAI retrieval. This study 


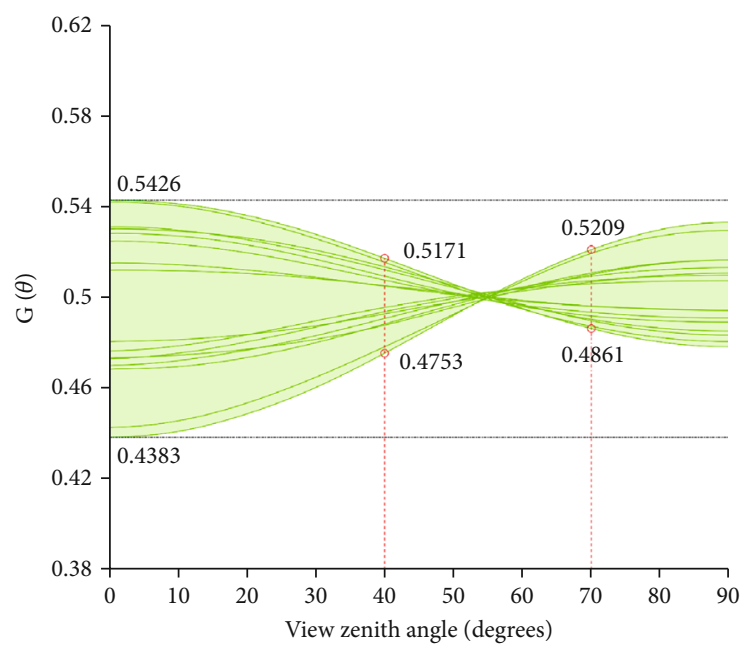

FIGURE 11: The G-functions computed from the rotated three-dimensional (3D) needle leaf models in Figure 9. The dark green line represents the measured G-function curve; the light-green region represents the range of G-function contained by two horizontal dashed lines; the red dashed lines show the range of view zenith angles from 40 to 70 degrees.

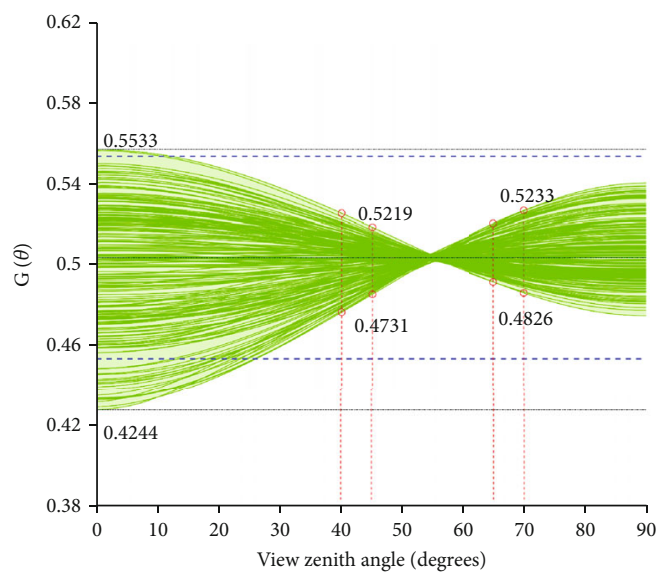

(a)

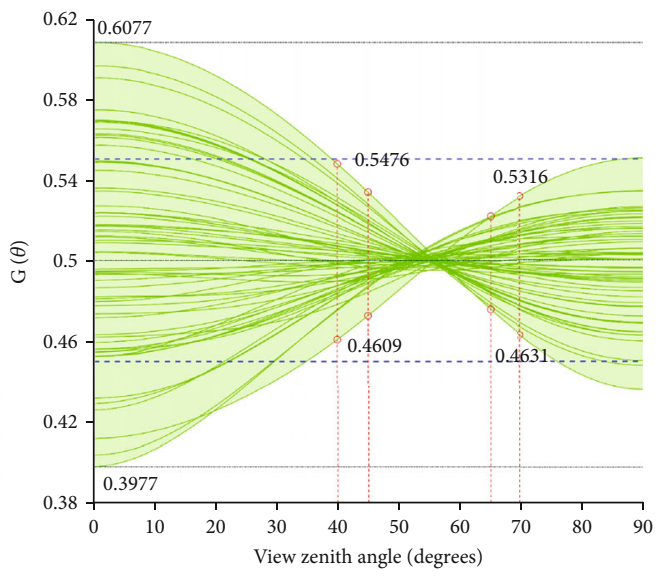

(b)

FIGURE 12: G-functions computed from the whole rotated three-dimensional (3D) needle leaf models: (a) Larch samples and (b) Spruce samples. The blue dashed lines represent $G=0.45$ and $G=0.55$, with a $\pm 10 \%$ deviation from $G=0.5$; the vertical red dashed lines show the range of G-function values for two brackets of view zenith angles of $\left(40^{\circ}, 70^{\circ}\right)$ and $\left(45^{\circ}, 65^{\circ}\right)$.

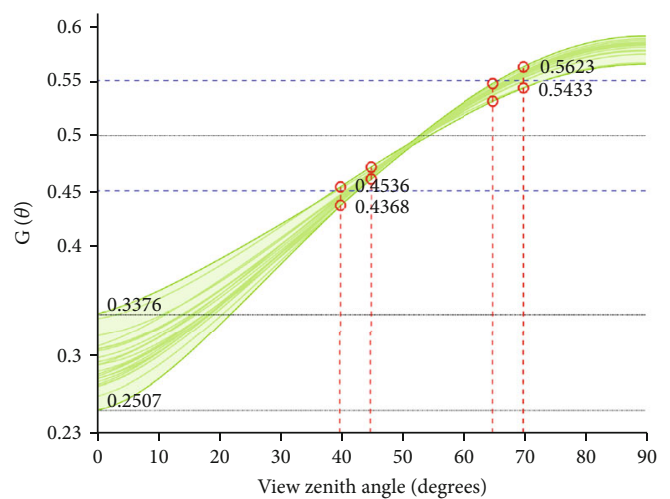

(a)

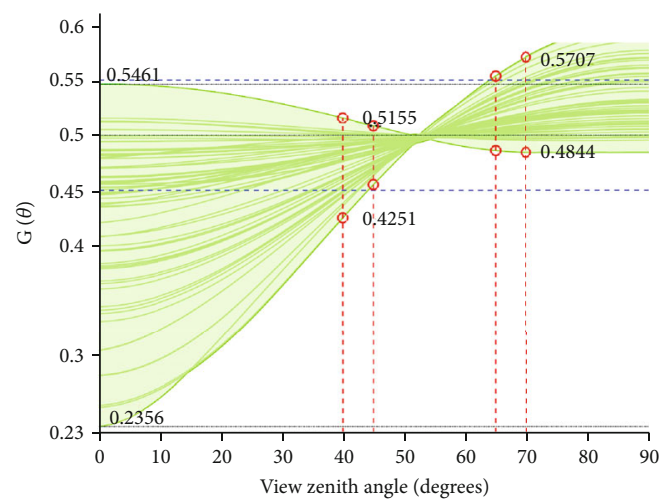

(b)

FIGURE 13: G-functions computed from inventory data and 3D tree models. (a) Inventory data of 23 trees of two tree species: Järvselja Scots Pine and Ofenpass Mountain Pine. (b) Three-dimensional (3D) tree models of 71 trees of 13 species (details in Table 3). The blue dashed lines represent $G=0.45$ and $G=0.55$, with a $\pm 10 \%$ deviation from $G=0.5$; the vertical red dashed lines show the range of $G$-function values for two brackets of view zenith angles of $\left(40^{\circ}, 70^{\circ}\right)$ and $\left(45^{\circ}, 65^{\circ}\right)$. 


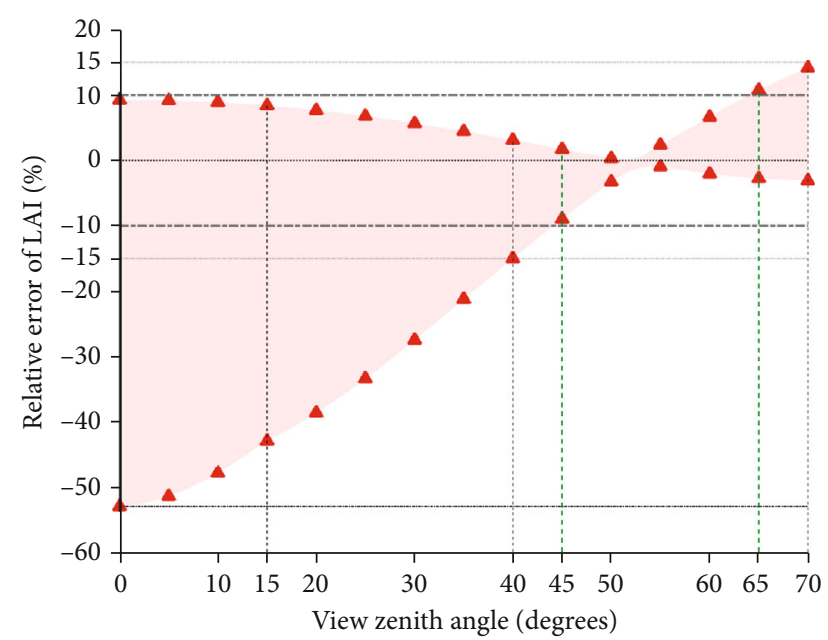

Figure 14: The range of relative errors of LAI (represented by the light-red region) with G-function set to 0.5 at different view zenith angles for all the studied species.

only focused on the impact of LAD on effective LAI. Previous studies showed that the clumping effect might cause a 30\% 70\% underestimation of true LAI $[42,57,58]$. Computer simulation, which can be used to generate diverse forest scenes with known gap fraction, LAD, and true LAI, might be a pathway to study the coupling effects of the clumping and the LAD to make sense of the total LAI retrieval error

\section{Conclusions}

Leaf inclination angle distribution (LAD) is an important factor affecting Beer's law-based LAI retrieval. Despite the high variability of LAD in reality, a spherical distribution assumption is often used for coniferous canopies due to the difficulties in measuring it. However, the error caused by this assumption has been seldom studied. We estimated the possible range of the G-function of two species by rotating the reconstructed needles of the branches measured by our developed equipment-MDI, which was an alternative to the manual measurement of the leaf inclination angle. To study more species, both 3D tree models and existing inventory data with known LADs were used. Results show that the range of the G-function is highly variable at different view zenith angles, indicating the necessity to measure LAD accurately to improve LAI retrieval. We found that the relative error of LAI with spherical distribution assumptions could be as large as $-53 \%$ in the zenithal view direction, which is nonnegligible for LAI retrieval from airborne and spaceborne LiDAR. Besides, our results show that $45^{\circ} \sim 65^{\circ}$ is a good range of the view zenith angle to reduce the impact of LAD on LAI retrieval (relative error less than 10\%) for coniferous canopies under the assumption of spherical distribution when the field-measured data is absent.

\section{Data Availability}

The data is freely available upon request.

\section{Additional Points}

General. Thank you for anonymous reviewers who gave constructive suggestions.

\section{Conflicts of Interest}

The authors declare no competing interests.

\section{Authors' Contributions}

G.Y. proposed the method and wrote the paper with J.L., H.J., and X.M. conceptualized the method and revised the paper. F.L., J.Q., and R.H. contributed to the field measurement, and D.X. and G.Z. revised the paper.

\section{Acknowledgments}

This work was supported by the key program of the National Natural Science Foundation of China (NSFC) (Grant No. 42090013); Guangxi Innovative Development Grand Grant under the grant number: Guike AA18118038; and the China Scholarship Council, Grant No. 201906040055.

\section{References}

[1] G. Yan, R. Hu, J. Luo et al., "Review of indirect optical measurements of leaf area index: recent advances, challenges, and perspectives," Agricultural and Forest Meteorology, vol. 265, pp. 390-411, 2019.

[2] G. Yan, R. Hu, Y. Wang et al., "Scale effect in indirect measurement of leaf area index," IEEE Transactions on Geoscience and Remote Sensing, vol. 54, no. 6, pp. 3475-3484, 2016.

[3] C. T. De Wit, "Photosynthesis of leaf canopies. Agri-cultural Research Report No 663," in Center for Agri-Cultural Publication and Documentation, pp. 1-57, Wagenin-gen, The Netherlands, 1965.

[4] J. Ross, The Radiation Regime and Architecture of Plant Stands, Springer, The Hague, Netherlands, 1981.

[5] H. Utsugi, M. Araki, T. Kawasaki, and M. Ishizuka, "Vertical distributions of leaf area and inclination angle, and their relationship in a 46-year-old Chamaecyparis obtusa stand," Forest Ecology and Management, vol. 225, no. 1-3, pp. 104-112, 2006.

[6] K. Omasa, F. Hosoi, and A. Konishi, "3D Lidar imaging for detecting and understanding plant responses and canopy structure," Journal of Experimental Botany, vol. 58, no. 4, pp. 881-898, 2006.

[7] F. Hosoi and K. Omasa, "Detecting seasonal change of broadleaved Woody canopy leaf area density profile using 3D portable Lidar imaging," Functional Plant Biology, vol. 36, no. 11, pp. 998-1005, 2009.

[8] K. F. Huemmrich, "Simulations of seasonal and latitudinal variations in leaf inclination angle distribution: implications for remote sensing," Advances in Remote Sensing, vol. 2, no. 2, pp. 93-101, 2013.

[9] K. Raabe, J. Pisek, O. Sonnentag, and K. Annuk, "Variations of leaf inclination angle distribution with height over the growing season and light exposure for eight broadleaf tree species," Agricultural and Forest Meteorology, vol. 214-215, pp. 2-11, 2015. 
[10] A. R. G. Lang, R. E. McMurtrie, and M. L. Benson, "Validity of surface area indices of Pinus radiata estimated from transmittance of the sun's beam," Agricultural and Forest Meteorology, vol. 57, no. 1-3, pp. 157-170, 1991.

[11] J. M. Chen and T. A. Black, "Foliage area and architecture of plant canopies from sunfleck size distributions," Agricultural and Forest Meteorology, vol. 60, no. 3-4, pp. 249-266, 1992.

[12] Y. Ryu, J. Verfaillie, C. Macfarlane et al., "Continuous observation of tree leaf area index at ecosystem scale using upwardpointing digital cameras," Remote Sensing of Environment, vol. 126, pp. 116-125, 2012.

[13] R. Hu, G. Yan, X. Mu, and J. Luo, "Indirect measurement of leaf area index on the basis of path length distribution," Remote Sensing of Environment, vol. 155, pp. 239-247, 2014.

[14] H. H. Neumann, G. Den Hartog, and R. H. Shaw, "Leaf area measurements based on hemispheric photographs and leaflitter collection in a deciduous forest during autumn leaf-fall," Agricultural and Forest Meteorology, vol. 45, no. 3-4, pp. 325345, 1989.

[15] S. G. Leblanc and J. M. Chen, "A practical scheme for correcting multiple scattering effects on optical LAI measurements," Agricultural and Forest Meteorology, vol. 110, no. 2, pp. 125139, 2001.

[16] D. L. B. Jupp, D. S. Culvenor, J. L. Lovell, G. J. Newnham, A. H. Strahler, and C. E. Woodcock, "Estimating forest LAI profiles and structural parameters using a ground-based laser called 'Echidna ${ }^{\circledR}, "$ Tree Physiology, vol. 29, no. 2, pp. 171-181, 2008.

[17] D. Culvenor, G. Newnham, A. Mellor, N. Sims, and A. Haywood, "Automated in-situ laser scanner for monitoring forest leaf area index," Sensors, vol. 14, no. 8, pp. 14994-15008, 2014.

[18] A. R. G. Lang, "Leaf orientation of a cotton plant," Agricultural Meteorology, vol. 11, pp. 37-51, 1973.

[19] H. Sinoquet and P. Rivet, "Measurement and visualization of the architecture of an adult tree based on a threedimensional digitising device," Trees, vol. 11, no. 5, pp. 265270, 1997.

[20] C. S. T. Daughtry, "Direct measurements of canopy structure," Remote Sensing Reviews, vol. 5, no. 1, pp. 45-60, 1990.

[21] B. N. Bailey and W. F. Mahaffee, "Rapid measurement of the three-dimensional distribution of leaf orientation and the leaf angle probability density function using terrestrial lidar scanning," Remote Sensing of Environment, vol. 194, pp. 63-76, 2017.

[22] Y. Ryu, O. Sonnentag, T. Nilson et al., "How to quantify tree leaf area index in an open savanna ecosystem: a multiinstrument and multi-model approach," Agricultural and Forest Meteorology, vol. 150, no. 1, pp. 63-76, 2010.

[23] J. Pisek, Y. Ryu, and K. Alikas, "Estimating leaf inclination and G-function from leveled digital camera photography in broadleaf canopies," Trees, vol. 25, no. 5, pp. 919-924, 2011.

[24] X. Zou, M. Mõttus, P. Tammeorg et al., "Photographic measurement of leaf angles in field crops," Agricultural and Forest Meteorology, vol. 184, pp. 137-146, 2014.

[25] B. E. McNeil, J. Pisek, H. Lepisk, and E. A. Flamenco, "Measuring leaf angle distribution in broadleaf canopies using UAVs," Agricultural and Forest Meteorology, vol. 218-219, pp. 204208, 2016.

[26] X. Li, J. Wang, and Y. Xiang, "Measuring leaf angle distribution and leaf area index of conifer canopies simultaneously on wide angle image," Journal of Remote Sensing, vol. 1, pp. 54-61, 1997.

[27] C. J. Kucharik, J. M. Norman, and S. T. Gower, "Measurements of leaf orientation, light distribution and sunlit leaf area in a boreal aspen forest," Agricultural and Forest Meteorology, vol. 91, no. 1-2, pp. 127-148, 1998.

[28] F. Hosoi, K. Nakabayashi, and K. Omasa, "3-D modeling of tomato canopies using a high-resolution portable scanning lidar for extracting structural information," Sensors, vol. 11, no. 2, pp. 2166-2174, 2011.

[29] K. Zhao, M. García, S. Liu et al., "Terrestrial lidar remote sensing of forests: maximum likelihood estimates of canopy profile, leaf area index, and leaf angle distribution," Agricultural and Forest Meteorology, vol. 209-210, pp. 100-113, 2015.

[30] F. Hosoi and K. Omasa, "Estimating leaf inclination angle distribution of broad-leaved trees in each part of the canopies by a high-resolution portable scanning lidar," Journal of Agricultural Meteorology, vol. 71, no. 2, pp. 136-141, 2015.

[31] G. Zheng and L. M. Moskal, "Leaf orientation retrieval from terrestrial laser scanning (TLS) data," IEEE Transactions on Geoscience and Remote Sensing, vol. 50, no. 10, pp. 39703979, 2012.

[32] L. Ma, G. Zheng, J. U. H. Eitel, T. S. Magney, and L. M. Moskal, "Retrieving forest canopy extinction coefficient from terrestrial and airborne lidar," Agricultural and Forest Meteorology, vol. 236, pp. 1-21, 2017.

[33] H. Wang, W. Zhang, G. Zhou, G. Yan, and N. Clinton, "Imagebased $3 \mathrm{D}$ corn reconstruction for retrieval of geometrical structural parameters," International Journal of Remote Sensing, vol. 30, no. 20, pp. 5505-5513, 2009.

[34] M. Müller-Linow, F. Pinto-Espinosa, H. Scharr, and U. Rascher, "The leaf angle distribution of natural plant populations: assessing the canopy with a novel software tool," Plant Methods, vol. 11, no. 1, p. 11, 2015.

[35] J. Qi, D. Xie, L. Li, W. Zhang, X. Mu, and G. Yan, "Estimating leaf angle distribution from smartphone photographs," IEEE Geoscience and Remote Sensing Letters, vol. 16, no. 8, pp. 1190-1194, 2019.

[36] C. Baillard, C. Schmid, A. Zisserman, and A. Fitzgibbon, "Automatic line matching and $3 \mathrm{D}$ reconstruction of buildings from multiple views," in Paper presented at the ISPRS Conference on Automatic Extraction of GIS Objects from Digital Imagery, Munich, Germany, 1999.

[37] A. Bartoli and P. Sturm, "Structure-from-motion using lines: representation, triangulation, and bundle adjustment," Computer Vision and Image Understanding, vol. 100, no. 3, pp. 416-441, 2005.

[38] H. Bay, A. Ess, A. Neubeck, and L. Van Gool, "3D from line segments in two poorly-textured, uncalibrated images," in Third International Symposium on 3D Data Processing, Visualization, and Transmission (3DPVT'06), pp. 496-503, Chapel Hill, NC, USA, 2006.

[39] L. Zhang and R. Koch, "Structure and motion from line correspondences: representation, projection, initialization and sparse bundle adjustment," Journal of Visual Communication and Image Representation, vol. 25, no. 5, pp. 904915, 2014.

[40] M. Rothermel, K. Wenzel, D. Fritsch, and N. Haala, "SURE: photogrammetric surface reconstruction from imagery," in Paper presented at the Proceedings LC3D Workshop, pp. 1-9, Berlin, Germany, 2012. 
[41] M. Hofer, M. Maurer, and H. Bischof, "Line3D: Efficient 3D Scene Abstraction for the Built Environment," in Paper presented at the Pattern Recognition, pp. 237-248, Springer, 2015.

[42] M. Weiss, F. Baret, G. J. Smith, I. Jonckheere, and P. Coppin, "Review of methods for in situ leaf area index (LAI) determination: Part II. Estimation of LAI, errors and sampling," Agricultural and Forest Meteorology, vol. 121, no. 1-2, pp. 37-53, 2004.

[43] X. Zhu, A. K. Skidmore, T. Wang et al., "Improving leaf area index (LAI) estimation by correcting for clumping and woody effects using terrestrial laser scanning," Agricultural and Forest Meteorology, vol. 263, pp. 276-286, 2018.

[44] L. Ma, G. Zheng, X. Wang, S. Li, Y. Lin, and W. Ju, “Retrieving forest canopy clumping index using terrestrial laser scanning data," Remote Sensing of Environment, vol. 210, pp. 452-472, 2018.

[45] J. Zou, G. Yan, L. Zhu, and W. Zhang, "Woody-to-total area ratio determination with a multispectral canopy imager," Tree Physiology, vol. 29, no. 8, pp. 1069-1080, 2009.

[46] R. G. von Gioi, J. Jakubowicz, J. M. Morel, and G. Randall, "LSD: a fast line segment detector with a false detection control," IEEE Transactions on Pattern Analysis and Machine Intelligence, vol. 32, no. 4, pp. 722-732, 2010.

[47] R. G. von Gioi, J. Jakubowicz, J.-M. Morel, and G. Randall, "LSD: a line segment detector," Image Processing On Line, vol. 2, pp. 35-55, 2012.

[48] J. B. Burns, A. R. Hanson, and E. M. Riseman, "Extracting straight lines," IEEE Transactions on Pattern Analysis and Machine Intelligence, vol. PAMI-8, no. 4, pp. 425-455, 1986.

[49] A. Desolneux, L. Moisan, and J.-M. Morel, "Meaningful alignments," International Journal of Computer Vision, vol. 40, no. 1, pp. 7-23, 2000.

[50] M. Hofer, M. Maurer, and H. Bischof, "Efficient 3D scene abstraction using line segments," Computer Vision and Image Understanding, vol. 157, pp. 167-178, 2017.

[51] P. Stenberg, "A note on the _G_-function for needle leaf canopies," Agricultural and Forest Meteorology, vol. 136, no. 1-2, pp. 76-79, 2006.

[52] J. L. Monteith, "Light distribution and photosynthesis in field crops," Annals of Botany, vol. 29, no. 1, pp. 17-37, 1965.

[53] J. Pisek, O. Sonnentag, A. D. Richardson, and M. Mõttus, "Is the spherical leaf inclination angle distribution a valid assumption for temperate and boreal broadleaf tree species?," Agricultural and Forest Meteorology, vol. 169, pp. 186-194, 2013.

[54] A. C. Brenner, H. J. Zwally, C. R. Bentley et al., "Derivation of range and range distributions from laser pulse waveform analysis for surface elevations," Roughness, Slope, and Vegetation Heights, Geoscience Laser Altimeter System Algorithm Theoretical Basis Document, Version 4, 2003, http://www.csr.utexas .edu/glas/pdf/Atbd_20031224.pdf.

[55] H. Tang and J. Armston, Algorithm Theoretical Basis Document (ATBD) for GEDI L2B Footprint Canopy Cover and Vertical Profile Metrics, Goddard Space Flight Center, Greenbelt, MD, USA, 2020, https://lpdaac.usgs.gov/documents/588/ GEDI_FCCVPM_ATBD_v1.0.pdf.

[56] R. Hu, G. Yan, F. Nerry et al., "Using airborne laser scanner and path length distribution model to quantify clumping effect and estimate leaf area index," IEEE Transactions on Geoscience and Remote Sensing, vol. 56, no. 6, pp. 3196-3209, 2018.
[57] J. M. Chen and J. Cihlar, "Plant canopy gap-size analysis theory for improving optical measurements of leaf-area index," Applied Optics, vol. 34, no. 27, pp. 6211-6222, 1995.

[58] P. Stenberg, "Correcting LAI-2000 estimates for the clumping of needles in shoots of conifers," Agricultural and Forest Meteorology, vol. 79, no. 1-2, pp. 1-8, 1996. 